\title{
Erratum: Fully Differential Vector-Boson-Fusion Higgs Production at Next-to-Next-to-Leading Order [Phys. Rev. Lett. 115, 082002 (2015)]
}

Matteo Cacciari, Frédéric A. Dreyer, Alexander Karlberg, Gavin P. Salam, and Giulia Zanderighi (Received 22 February 2018; published 27 March 2018)

DOI: 10.1103/PhysRevLett.120.139901

The NLO 3-jet calculation [1] and its POWHEG implementation [2] has been found to have a bug in its virtual terms. Resolving this bug reduces the impact of the NNLO corrections and they are now of order $4 \%$ for the total cross section (with VBF cuts) and up to 4\%-7\% in differential distributions. Updated results for the total cross section and differential distributions are to be found in Table I and Fig. 1, respectively.

TABLE I. Cross sections at LO, NLO, and NNLO for VBF Higgs production, fully inclusively and with VBF cuts. The quoted uncertainties correspond to scale dependence, while statistical errors at NNLO are about $0.1 \%$ with VBF cuts and much smaller without.

\begin{tabular}{lcr}
\hline \hline & $\sigma^{(\text {no cuts })}[\mathrm{pb}]$ & $\sigma^{(\mathrm{VBF} \text { cuts })}[\mathrm{pb}]$ \\
\hline LO & $4.032_{-0.069}^{+0.057}$ & $0.957_{-0.059}^{+0.066}$ \\
NLO & $3.929_{-0.023}^{+0.024}$ & $0.876_{-0.018}^{+0.008}$ \\
NNLO & $3.888_{-0.012}^{+0.016}$ & $0.844_{-0.008}^{+0.008}$ \\
\hline \hline
\end{tabular}
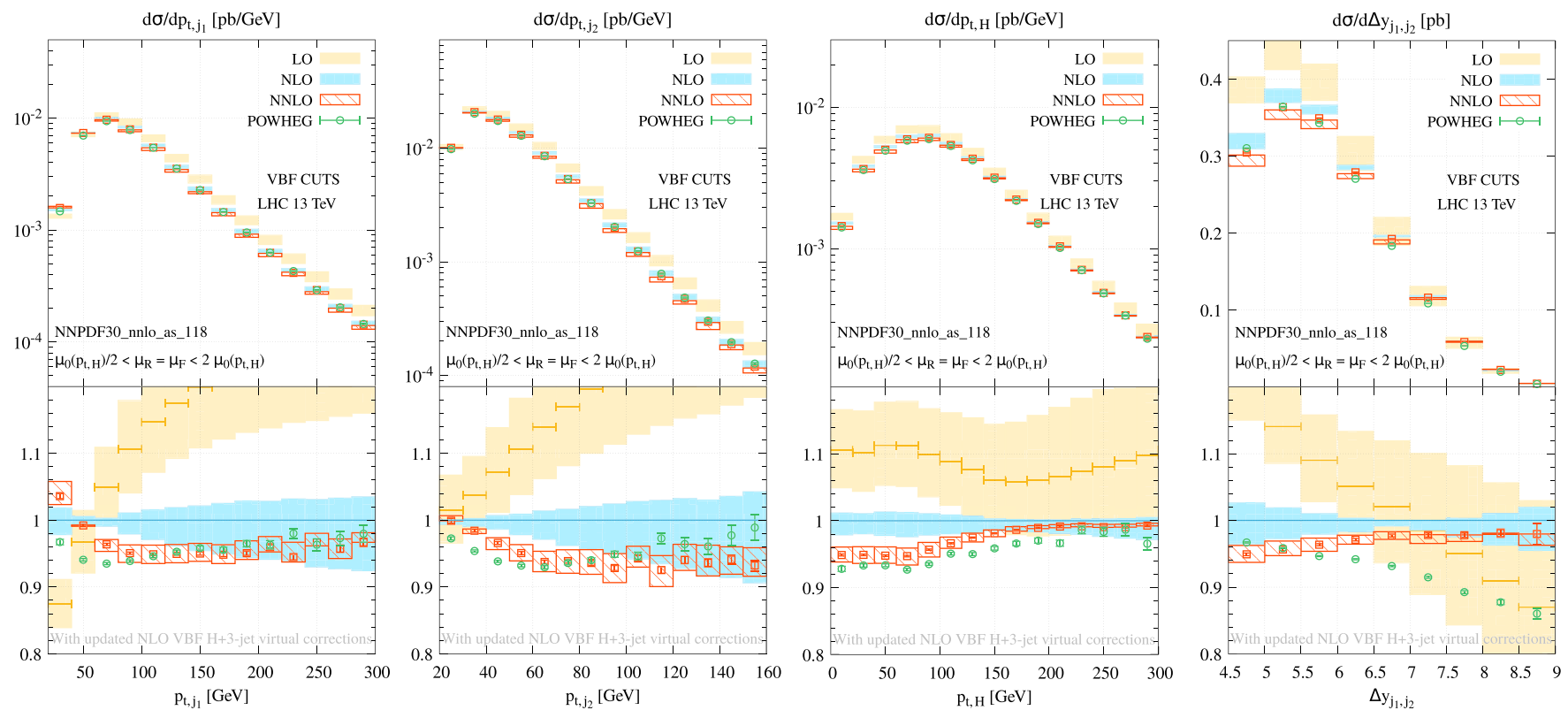

FIG. 1. From left to right, differential cross sections for the transverse momentum distributions for the two leading jets, $p_{t, j_{1}}$ and $p_{t, j_{2}}$, for the Higgs boson, $p_{t, H}$, and the distribution for the rapidity separation between the two leading jets, $\Delta y_{j_{1}, j_{2}}$.

Published by the American Physical Society under the terms of the Creative Commons Attribution 3.0 License. Further distribution of this work must maintain attribution to the author(s) and the published articles title, journal citation, and DOI. 
We are grateful to the authors of Ref. [3], as well as Barbara Jäger, for bringing the bug to our attention and to the latter for providing an associated fix. We also thank the authors of Ref. [3] for detailed comparisons with their results prior to publication.

[1] T. Figy, V. Hankele, and D. Zeppenfeld, J. High Energy Phys. 02 (2008) 076.

[2] B. Jäger, F. Schissler, and D. Zeppenfeld, J. High Energy Phys. 07 (2014) 125.

[3] J. Cruz-Martinez, T. Gehrmann, E. W. N. Glover, and A. Huss, arXiv:1802.02445. 